\title{
Effects of domains in phonon conduction through hybrid boron nitride and graphene sheets
}

\author{
H. Sevinçli, ${ }^{1,{ }^{*}}$ W. Li,,${ }^{1,2,3,{ }^{*}}$ N. Mingo, ${ }^{3}$ G. Cuniberti, ${ }^{1,4}$ and S. Roche ${ }^{1,5,6}$ \\ ${ }^{1}$ Institute for Materials Science and Max Bergmann Center of Biomaterials, Dresden University of Technology, DE-01062 Dresden, Germany \\ ${ }^{2}$ Institute of Physics, Chinese Academy of Sciences, CN-100190 Beijing, China \\ ${ }^{3}$ LITEN, CEA, 17 rue des Martyrs, BP 166, FR-38042 Grenoble, France \\ ${ }^{4}$ Division of IT Convergence Engineering, POSTECH, Pohang KR-790-784, Republic of Korea \\ ${ }^{5}$ CIN2 (ICN-CSIC) and Universitat Autónoma de Barcelona, Catalan Institute of Nanotechnology, Campus UAB, ES-08193 Bellaterra \\ (Barcelona), Spain \\ ${ }^{6}$ ICREA, Institució Catalana de Recerca i Estudis Avancats, ES-08070 Barcelona, Spain
}

(Received 14 June 2011; revised manuscript received 7 September 2011; published 21 November 2011)

\begin{abstract}
We theoretically investigate the phonon propagation and thermal conductivity $\kappa$ in hybrid boron nitride and graphene sheets. By using a real-space Kubo-computational transport scheme, large and disordered graphene structures are simulated, introducing disk-shaped domains with varying sizes of 2 to $8 \mathrm{~nm}$ and concentrations ranging from $0 \%$ to $100 \%$. A strong influence of the domain size and concentration on the transport properties is obtained. The mean free paths are minimized at $50 \%$ domain concentration, and stronger suppression of $\kappa$ is achieved with smaller domains. It is found to decrease by up to $65 \%$ at room temperature when the domain size is $2 \mathrm{~nm}$. These results are beyond the scope of any effective medium approximation.
\end{abstract}

DOI: 10.1103/PhysRevB.84.205444

PACS number(s): 73.22.Pr, 72.80.Vp, 72.15.Rn

\section{INTRODUCTION}

There is growing interest in two-dimensional (2D) materials for nanoelectronics applications. In this respect, graphene $e^{1,2}$ and BN monolayers ${ }^{3-5}$ are sources of exciting new physics. ${ }^{6,7}$ The number of materials that have been exfoliated as single layers keeps increasing as shown very recently by the cases of $\mathrm{Bi}_{2} \mathrm{Te}_{3}{ }^{8}$ and $\mathrm{MoS}_{2} .{ }^{9}$ In parallel with their syntheses, the electronic and thermal conduction properties of these materials are the target of very recent theoretical and experimental investigations, and they continue to yield surprises. ${ }^{10-18}$

An exciting possibility has been triggered by the demonstration of domain formation in these layers. For example, synthesis of isotopic graphene domains was reported, ${ }^{19}$ and hybrid BN-C domains were evidenced. ${ }^{20,21}$ The possible impact of isotopic domains on thermal conduction was suggested in Ref. 22, which predicted a strong size dependence of the thermal conductivity of graphene when small isotopic ${ }^{13} \mathrm{C}$ clusters were embedded in the ${ }^{12} \mathrm{C}$ graphene host. That study was based on the assumption that each cluster scatters phonons independently and that multiple-scattering effects can be ignored, an approach dating back to the effective-medium virtual crystal approximation (VCA), already employed by Abeles in his analysis of alloy thermal conductivity. ${ }^{23}$ This is reasonable for small isotopic concentrations, but it becomes questionable whether such a picture holds for intermediate concentrations far from $0 \%$ or $100 \%$. Thus, in order to tackle the problem at any concentration without resorting to any approximations, we have implemented an order- $N$ real-space Kubo formalism, ${ }^{24,25}$ which allows us to simulate large systems.

In this work, we investigate the effects of domains on phonon transport through hybrid $\mathrm{BN}$-graphene sheets as illustrated in Fig. 1. The remainder of this paper is structured as follows. In Sec. II, we briefly introduce the real-space Kubo method. In Sec. III, we investigate the effects of the BN concentration, size of the domains, and B isotopes on thermal conductivity. Finally, we present our conclusions in Sec. IV.

\section{METHODOLOGY}

In the real-space Kubo approach, ${ }^{24,25}$ we calculate the time-dependent diffusivity $\mathcal{D}(\omega, t)=\chi^{2}(\omega, t) / t$, where $\chi^{2}$ is the mean-square spreading of the wave packets of frequency $\omega$ along the transport direction $x$. D behaves as $v_{x}^{2} t$ for short times, where $v_{x}$ is the average velocity along the $x$ direction. It saturates to $\mathcal{D}_{\max } \approx 2 v_{x}^{2} \tau_{\text {tr }}$ in the diffusive regime, where $\tau_{\text {tr }}$ is the average transport time, and then decreases due to localization effects. Considering $v_{x}^{2}=v^{2} / 2$ in two dimensional systems, the mean free path (MFP) can be obtained via $\ell(\omega)=\mathcal{D}_{\max }(\omega) / \sqrt{2} v_{x}(\omega)$. Mean-square displacement can be expressed as

$$
\chi^{2}(\omega, t)=\frac{\operatorname{Tr}\left\{[X, U(t)]^{\dagger} \delta\left(\omega^{2}-D\right)[X, U(t)]\right\}}{\operatorname{Tr}\left[\delta\left(\omega^{2}-D\right)\right]},
$$

where $X$ is the position matrix, $D$ is the mass-normalized dynamical matrix, and $U(t)=e^{-i \sqrt{D} t}$ serves as the evolution operator. The trace in Eq. (1) can be efficiently calculated through an average over a few initial random-phase states and can be further evaluated by using a Chebyshev expansion of $U(t)$ and the Lanczos method. It yields a mode-averaged value for the mean-square displacement and the mean free path. Possible shortcomings due to averaging over decoupled modes are avoided as explained below.

The fourth-nearest-neighbor cutoff is proven to describe phonons of graphene accurately, ${ }^{22}$ and the fourth-nearestneighbor force-constant (4NNFC) scheme allows a fairly accurate description of phonon spectra and heat transport properties of $s p^{2}$-hybrid carbon systems. ${ }^{26,27}$ We use the 4NNFC parameters from the pure graphene system ${ }^{27}$ and treat $\mathrm{B}$ and $\mathrm{N}$ atoms in terms of their mass differences as an approximation. In Fig. 2, phonon dispersions of single-layer BN obtained with the 4NNFC-mass-difference approximation are compared with those obtained from Tersoff-type parametrizations, ${ }^{28}$ density functional theory calculations, ${ }^{28}$ and experiments on bulk hexagonal $\mathrm{BN} .^{29}$ It is observed that DFT calculations 


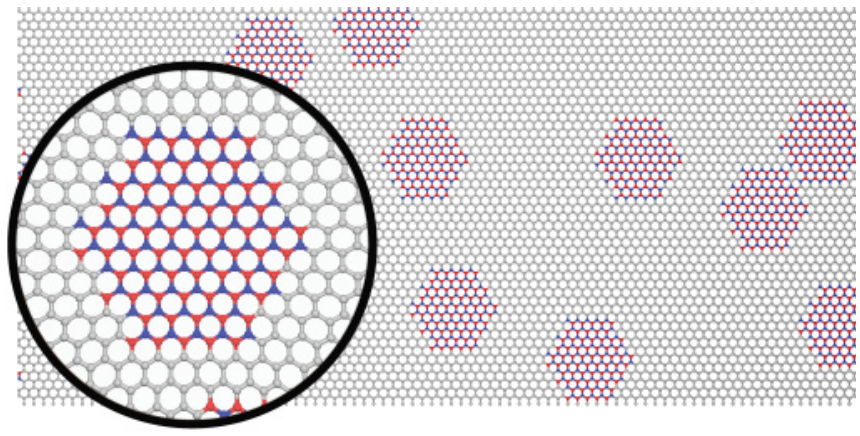

FIG. 1. (Color online) A schematic representation of the atomic structure of two-dimensional graphene-BN heterostructures. Here, $\mathrm{BN}$ clusters with 2-nm diameters are embedded inside the graphene.

yield the best agreement with the experiments. LA and TA modes of the 4NNFC approach are in very good agreement with the DFT results and the experiments. The ZO mode obtained from the 4NNFC approach agrees fairly well with the DFT results, and it gives a better estimation than the Tersoff parametrization. On the other hand, the 4NNFC approach cannot predict the ZA mode as well as it does the LA, $\mathrm{TA}$, and $\mathrm{ZO}$ modes. It overestimates the group velocities of ZA phonons and underestimates the out-of-plane energy gap, namely, the ZA-ZO splitting around $K$. The 4NNFC approach underestimates the group velocities of the $\mathrm{LO}$ and TO modes, but these high-energy modes have only a minor contribution to the room-temperature thermal conductivity.

Comparing the 4NNFC approach with the Tersoff parametrization, we find that the LA and TA modes are equally well described by both methods. The ZO mode is better described by the 4NNFC approach while the Tersoff parametrization more accurately predicts the ZA mode. While both the 4NNFC and Tersoff approaches underestimate the ZA-ZO splitting around $K$, the 4NNFC method gives a better estimation of the group velocities of these phonons. The maximum and minimum frequencies of the in-plane optical

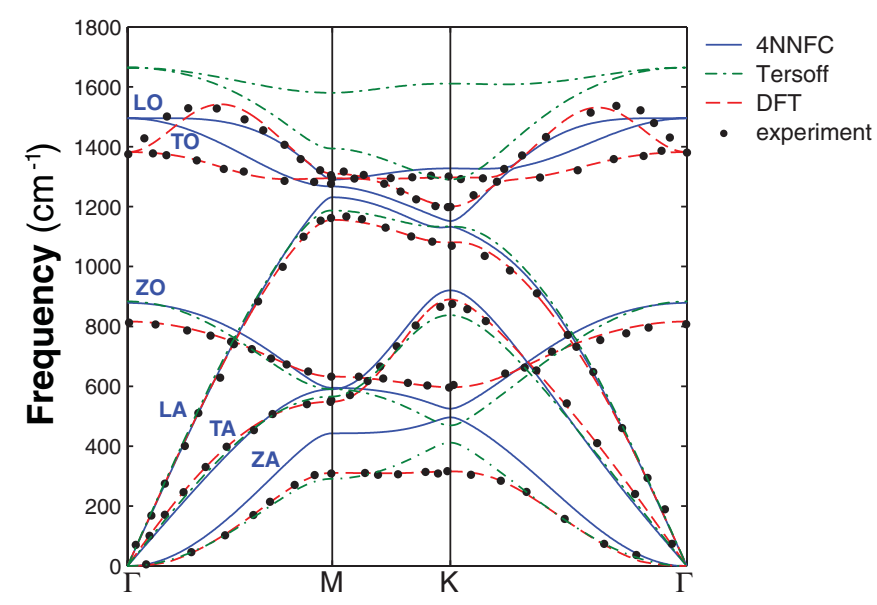

FIG. 2. (Color online) Comparison of the phonon dispersions of a single sheet of $\mathrm{BN}$ obtained from the fourth-nearest-neighbor forceconstant model with Tersoff-type parametrization (data taken from Ref. 28) and density-functional-theory calculations. Experimental results are also included for comparison. modes (LO and TO) are estimated correctly with the 4NNFC approach while the Tersoff parametrization overestimates them.

In summary, the in-plane modes LA, TA, TO, and LO are well described by the 4NNFC mass-difference approximation. Considering the phonon populations at room temperature, we conclude that their contribution to thermal conductivity is accurately estimated by the 4NNFC approach. The out-ofplane ZA and ZO modes are not predicted as well as the inplane modes. Experimental results and DFT calculations show that the out-of-plane modes display a gap in the $350-600 \mathrm{~cm}^{-1}$ frequency window. In parallel to this, we estimate that the shortest mean free paths in order of $10 \mathrm{~nm}$ or below lie within the $450-600 \mathrm{~cm}^{-1}$ region. Since the simulation window's size is $1 \mu \mathrm{m} \times 1 \mu \mathrm{m}$, we predict a transport gap at these energies. Therefore, we conclude that the 4NNFC mass-difference approach reproduces thermal transport properties of hybrid $\mathrm{BN}$-graphene heterostructures satisfactorily. ${ }^{28-30}$

The quadratic out-of-plane vibrational modes are completely decoupled from the in-plane modes, ${ }^{27}$ so we can study the MFPs separately, simply by limiting the initial random-phase state to out-of-plane vibrations or in-plane vibrations. This also enables us to distinguish the fundamental differences between in-plane and out-of-plane vibrations. The system size is chosen to be $100 \mathrm{~nm} \times 100 \mathrm{~nm}$, and periodic boundary conditions are employed. It is known that the MFP diverges as $\omega \rightarrow 0$. Ideally, one needs to consider an infinite system to be able to calculate MFPs for the entire spectrum, which is practically impossible. On the other hand, below a frequency threshold the MFP becomes comparable with that of our assumed sample size $(1 \mu \mathrm{m} \times 1 \mu \mathrm{m})$, so the effect of domains at these frequencies is masked by the sample boundary scattering. Accordingly, we calculate the MFP down to $50 \mathrm{~cm}^{-1}$, and intrinsic scattering is neglected below this frequency.

\section{RESULTS}

We investigate the effect of the overall concentration of BN as well as the influence of the domain size on the mean free path in the cases with pure and isotopic mixtures of boron. In our simulations the domains are disk-shaped, and their diameters are set to be 2, 4, or $8 \mathrm{~nm}$ (Fig. 1).

\section{A. Concentration effect}

In studying the effects of concentration, domain diameter is fixed to be $2 \mathrm{~nm}$ with a single isotopic species of boron, namely ${ }^{11} \mathrm{~B}$. We consider five different atomic concentrations of BN, $20 \%, 40 \%, 50 \%, 60 \%$, and $80 \%$, while the ratio of B and $\mathrm{N}$ is $1: 1$ in all cases. For the concentrations $20 \%$ and $40 \%$, the host matrix of the sheet is graphene while $\mathrm{BN}$ is considered to be the host matrix for the $60 \%$ and $80 \%$ concentrations. For the $50 \%$ concentration, both of the matrices are considered. Our calculations show that different choices of the host give similar results owing to disorder average.

The calculated transport MFPs are plotted in Fig. 3. The density of states (DOS) is very low around $537 \mathrm{~cm}^{-1}$ for graphene, corresponding to the intersection of the dispersions of the ZA and ZO modes at the $K$ point of the Brillouin 


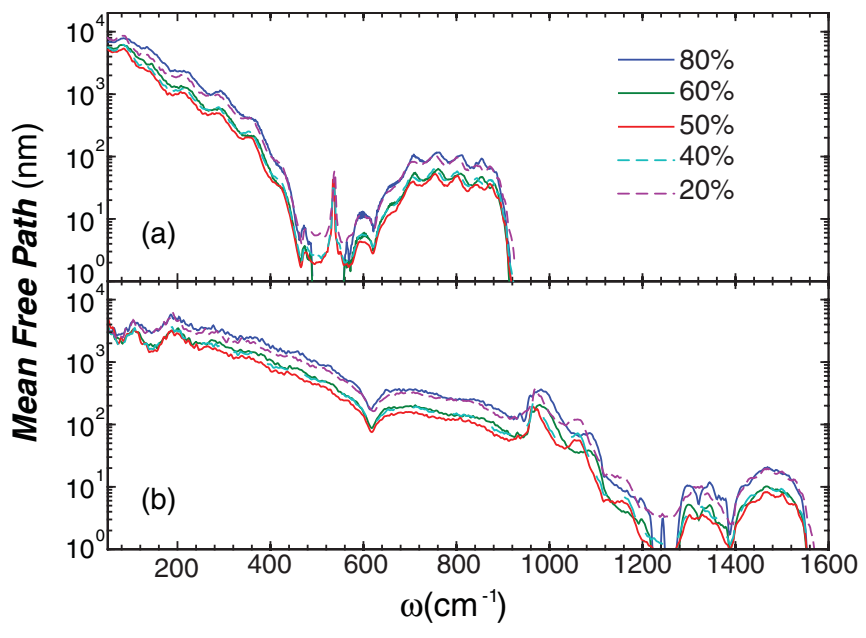

FIG. 3. (Color online) Mean free paths for different concentrations at a fixed domain size of $2 \mathrm{~nm}$ are plotted for (a) out-of-plane and (b) in-plane modes, separately.

zone. In the case of a pure BN sheet, these modes do not intersect but repel each other due to broken-sublattice symmetry, and the DOS is even lower than that of graphene. ${ }^{31}$ As a result, out-of-plane modes are suppressed very strongly at these energies as the BN concentration is increased. In order to gain more insight, we also performed calculations with random distributions of individual $\mathrm{C}$ atoms in $\mathrm{BN}$ hosts (Fig. 4). In this case, the MFPs decrease with increasing C concentrations for both in-plane and out-of-plane modes, the minimum value being achieved at a $50 \%$ concentration. We note that at lower frequencies, out-of-plane modes have shorter MFPs for the atomic distributions while for in-plane modes, cluster distributions have shorter MFPs below $100 \mathrm{~cm}^{-1}$. An interesting feature of the cluster distributions becomes more apparent upon comparison with the atomic distributions, namely, the oscillations in the MFP as a function of frequency are characteristic of the clustering. VCA predicts a quadratic dependence of the MFP on the density within the massdifference approximation $\ell^{-1} \sim d(1-d)$. Our results are in

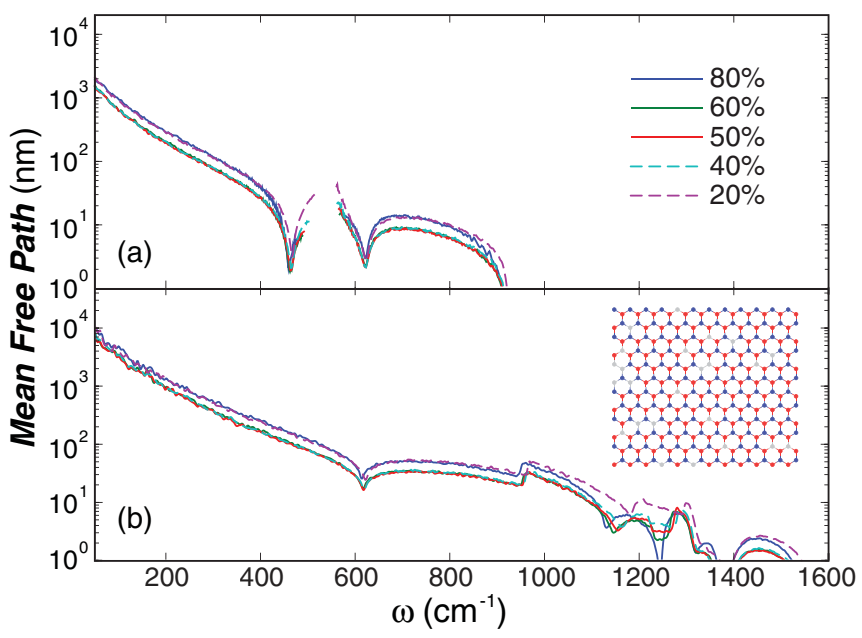

FIG. 4. (Color online) $\mathrm{C}$ atoms randomly distributed in the $\mathrm{BN}$ host (see the illustration in the inset). MFPs with different concentrations of $\mathrm{C}$ atoms for (a) out-of-plane and (b) in-plane modes. agreement with the prediction that the minimum MFP is achieved at $d=0.5$, but simple quadratic density dependence is not reproduced by our calculations in which $\lambda_{d=0.5} / \lambda_{d \neq 0.5}$ is generally smaller than that of the VCA prediction as expected due to the incorporation of multiple-scattering effects in our calculations.

\section{B. Domain-size effect}

In order to investigate the influence of the domain sizes, we keep the concentration fixed at $50 \%$ and generate random samples with domains having diameters $d=2,4$, or $8 \mathrm{~nm}$. The host is graphene, and all $\mathrm{B}$ atoms are of the same isotopic species, ${ }^{11} \mathrm{~B}$. We observe a general increase in the MFPs with increasing domain size (Fig. 5). In the long wave limit $(\omega \rightarrow 0)$, MFPs should decrease with increasing size; ${ }^{22,32}$ therefore, at very low temperatures, thermal conductivity can be reduced by increasing the domain size. However, the MFPs at these low frequencies become comparable to our chosen sample size of $1 \mu \mathrm{m}$. We also compare the MFPs due to disk-shaped domains with that due to hexagonal scatterers consisting of only 6 atoms where the diameter is $\simeq 0.3 \mathrm{~nm}$. For $\omega>200 \mathrm{~cm}^{-1}$, MFPs due to hexagonal scatterers are generally smaller. At $\omega<200 \mathrm{~cm}^{-1}$, the crossover takes place for the in-plane modes, and long-wavelength phonons are scattered less effectively by the small-diameter hexagonal domains. At the other extreme for $\omega>1300 \mathrm{~cm}^{-1}$, hexagons give rise to very short localization lengths due to vanishingly small MFPs. The domains considered here are considerably larger than those in Ref. 22. For these large sizes and frequencies down to $200 \mathrm{~cm}^{-1}$, scattering is in the short-wave regime, characterized by MFPs proportional to the domain diameters. This is evident from the overlapping curves of the plots of $\ell / d(d=2$, $4,8 \mathrm{~nm}$ ). In contrast, in the long-wavelength, or Rayleigh, regime, the MFP for a fixed-domain surface fraction in two dimensions would be inversely proportional to the square of the domain size. Rayleigh theory predicts the cross section of a cluster to be proportional to the square of the number

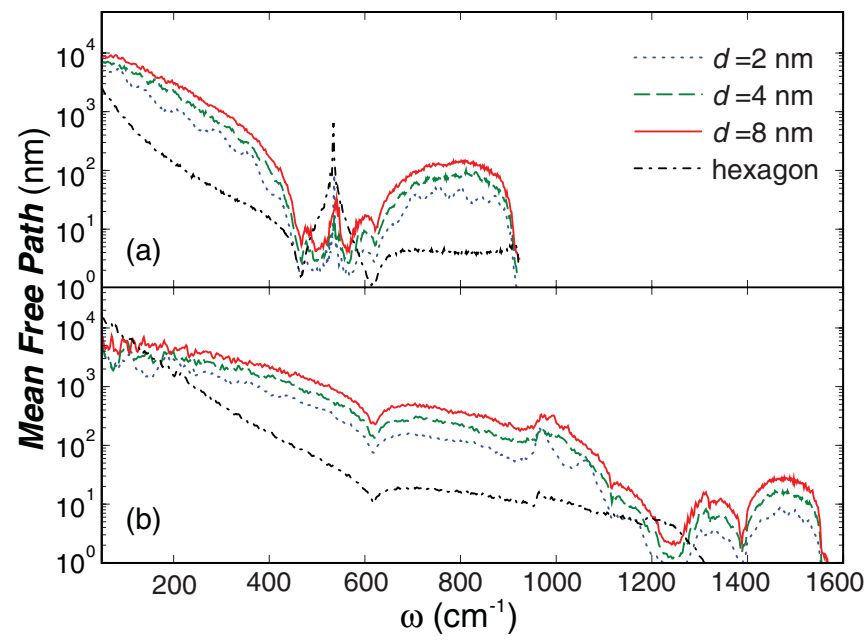

FIG. 5. (Color online) MFPs for different domain sizes at a fixed concentration of $50 \%$ for (a) out-of-plane and (b) in-plane modes. MFPs with domains consisting of single hexagons are also plotted for comparison. 


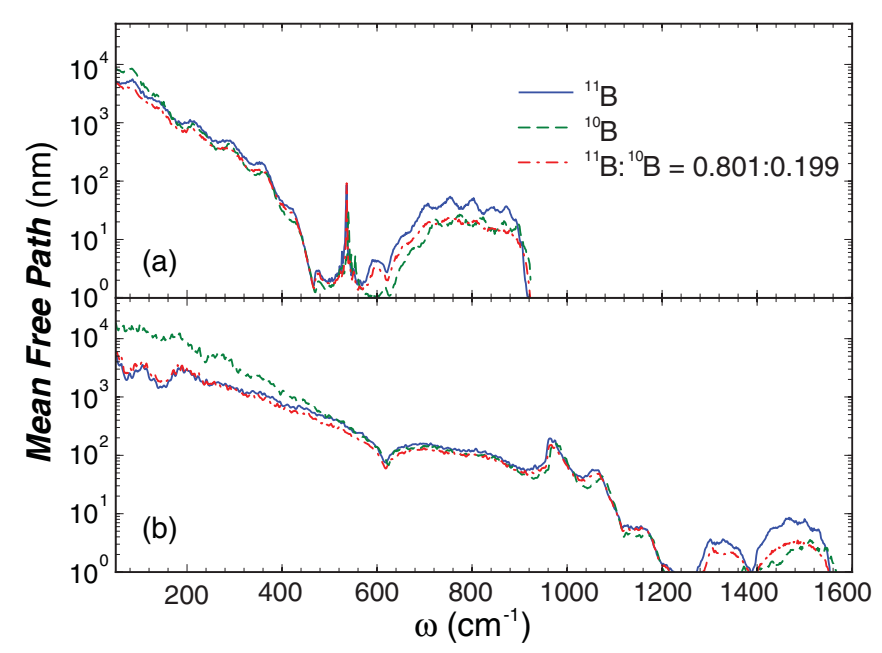

FIG. 6. (Color online) MFPs for different B isotopic compositions at a $50 \%$ concentration and 2-nm domain size. (a) Out-of-plane and (b) in-plane modes.

of atoms in the cluster at low frequencies. Our calculations confirm that scattering per atom is stronger for large clusters at low frequencies for which the crossover frequency is around $200 \mathrm{~cm}^{-1}$. The MFPs of quadratic out-of-plane modes show an $\omega^{-3 / 2}$ dependence at low frequencies as predicted by Rayleigh scattering theory ${ }^{22}$ while those of linear in-plane modes display an $\omega^{-3}$ dependence.

\section{Isotope effect}

We now study the effect of isotopic distribution on a graphene host with a $50 \%$ BN concentration, keeping the domain size fixed as $d=2 \mathrm{~nm}$. Boron has two stable isotopes, ${ }^{11} \mathrm{~B}$ and ${ }^{10} \mathrm{~B}$ with natural abundances of $80.1 \%$ and $19.9 \%$, respectively. Three different $\mathrm{B}$ compositions are considered: pure ${ }^{11} \mathrm{~B}$, pure ${ }^{10} \mathrm{~B}$, and a mixture of the two with their natural abundances. For isotopically pure ${ }^{10} \mathrm{~B}$, the average mass of a $\mathrm{BN}$ cluster is approximately equal to that of graphene while it is larger for pure ${ }^{11} \mathrm{~B}$. As shown in Fig. 6, MFPs for the pure ${ }^{10} \mathrm{~B}$ case are longer than those for the pure ${ }^{11} \mathrm{~B}$ case at low frequencies. Though the mass difference between ${ }^{10} \mathrm{~B}$ and ${ }^{12} \mathrm{C}$ is larger than the difference between ${ }^{11} \mathrm{~B}$ and ${ }^{12} \mathrm{C}$, the negative mass difference is canceled out by the positive mass difference between ${ }^{14} \mathrm{~N}$ and ${ }^{12} \mathrm{C}$, leading to stronger scattering by ${ }^{11} \mathrm{~B}$ than by ${ }^{10} \mathrm{~B}$ at low frequencies. In other words, low-frequency modes are more sensitive to the average mass of the BN clusters while at high frequencies, the deviation of atomic masses from the average are more effective. One also observes that the curves for the mixture case do not simply fall in between the curves of the isotopically pure cases. At low frequencies, they follow the ${ }^{11} \mathrm{~B}$ curve, which is more abundant, but at higher frequencies, this tendency is lost.

\section{Thermal conductivity}

In a sample of length $L$ with perfect contacts, $\kappa$ can be approximated as ${ }^{33}$

$$
\kappa_{o / i}=\frac{1}{h} \int_{0}^{\infty} \frac{d \omega}{2 \pi} \hbar \omega \frac{\partial f}{\partial T}\left(1+\frac{4}{\pi} \frac{L}{2 \ell_{o / i}(\omega)}\right)^{-1} \mathcal{T}_{o / i}(\omega)
$$

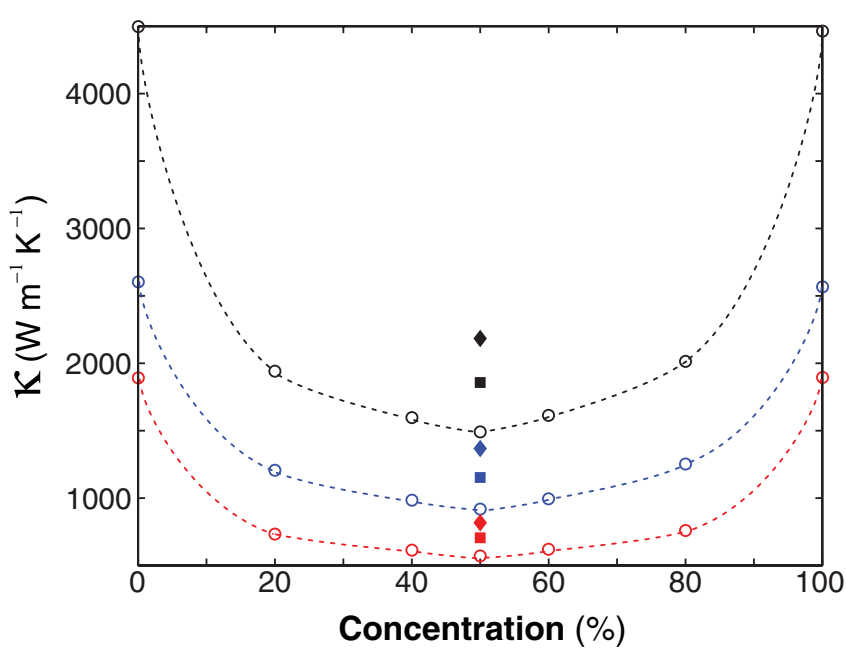

FIG. 7. (Color online) Room-temperature thermal conductivity for a 2-nm domain size versus the concentration of BN contributed by out-of-plane modes (blue/dark gray), in-plane modes (red/gray), and all modes (black). Dashed curves are plotted as guides to the eye. At $50 \%$ concentration, two additional domain sizes are considered. Circles: $2 \mathrm{~nm}$; squares: $4 \mathrm{~nm}$; and diamonds: $8 \mathrm{~nm}$.

assuming that the phonon dispersions and mean free paths are isotropic. This approximation becomes more accurate as the scattering rates are increased and the modes are mixed more effectively. In Eq. (2), $i$ and $o$ denote in-plane and out-of-plane modes, respectively. $h=3.35 \AA$ is the graphitic interlayer spacing, $f$ is the Bose function, and $\mathcal{T}$ is the ballistic transmission, which is computed from the phonon dispersions in the entire Brillouin zone. For $\omega<50 \mathrm{~cm}^{-1}$, phonons are assumed to propagate ballistically, and the term in the brace is approximated by 1 (see Sec. II). The calculated conductivities for a 2-nm domain size for different concentrations are plotted in Fig. 7. Conductivities for $d=4$ and $8 \mathrm{~nm}$ are also indicated for a $50 \%$ concentration. Conductivity changes rapidly near $0 \%$ and $100 \%$ concentrations, but this change is considerably slower around a $50 \%$ concentration. The conductivity is nearly symmetric around a $50 \%$ concentration with a minimum where it is reduced by $65 \%$, compared to pure graphene. Such symmetric behavior could be altered with a more accurate description of the out-of-plane vibrations, but we do not expect a major change. A considerable increase in the conductivity can be observed when the domain size increases. A cautionary note is in order here. Since we do not include the effect of anharmonicity, the conductivity of pure graphene depends linearly on $L$. In contrast, the conductivities of the hybriddomain systems are nearly independent of length. Therefore, the obtained reduction in the thermal conductivity also depends on the sample's length.

\section{CONCLUSIONS}

Using a real-space method to compute phonon propagation, we have reported the strong effects of domains on thermal conductivity and have shown that hybrid BN-C layers have interesting possibilities regarding the tunability of their thermal conductivities by simultaneously adjusting the domain size and concentration. Suppression of the thermal 
conductivity is less pronounced with larger domains while a $65 \%$ reduction in room-temperature thermal conductivity is possible when the clusters have 2-nm diameters and a $50 \%$ concentration.

Note added in proof. Another set of parameters has been recently derived for hexagonal $\mathrm{BN}$ interatomic potentials and applied to study its thermal conductivity in Ref. 34 .

\section{ACKNOWLEDGMENTS}

We would like to acknowledge support from the priority program Nanostructured Thermoelectrics (SPP-1386) of the German Research Foundation (DFG) (Contract No. CU 44/11$1)$, the cluster of excellence of the Free State of Saxony
ECEMP-European Center for Emerging Materials and Processes Dresden (Project A2), the European Social Funds in Saxony (research group InnovaSens), the ANR/P3N2009 NANOSIM-GRAPHENE Project No. ANR-09-NANO-01601, and the Alexander von Humboldt Foundation. W.L. thanks the CAS-MPG joint doctoral promotion program. N.M. acknowledges support from Fondation Nanosciences and Agence Nationale de la Recherche (ANR) and from CEA through project THERMA. G.C. further acknowledges the World Class University program sponsored by the South Korean Ministry of Education, Science, and Technology Program, Project No. R31-2008-000-10100-0. We also acknowledge the Center for Information Services and High Performance Computing $(\mathrm{ZIH})$ at the TU Dresden.
*The first two authors contributed equally and share first authorship of this work.

${ }^{1}$ K. S. Novoselov, A. K. Geim, S. V. Morozov, D. Jiang, Y. Zhang, S. V. Dubonos, I. V. Grigorieva, and A. A. Firsov, Science 306, 666 (2004).

${ }^{2}$ A. Cresti, N. Nemec, B. Biel, G. Niebler, F. Triozon, G. Cuniberti, and S. Roche, Nano Res. 1, 361 (2008).

${ }^{3}$ K. S. Novoselov, D. Jiang, F. Schedin, T. J. Booth, V. V. Khotkevich, S. V. Morozov, and A. K. Geim, Proc. Natl. Acad. Sci. USA 102, 10451 (2005).

${ }^{4}$ A. Nagashima, N. Tejima, Y. Gamou, T. Kawai, and C. Oshima, Phys. Rev. Lett. 75, 3918 (1995).

${ }^{5}$ D. Pacile, J. C. Meyer, C. O. Girit, and A. Zettl, App. Phys. Lett. 92, 133107 (2008).

${ }^{6}$ A. Ramasubramaniam and D. Naveh, Phys. Rev. B 84, 075405 (2011).

${ }^{7}$ A. Rubio, Nat. Mater. 9, 379 (2010).

${ }^{8}$ D. Teweldebrhan, V. Goyal, M. Rahman, and A. A. Balandin, App. Phys. Lett. 96, 053107 (2010).

${ }^{9}$ B. Radisavljevic, A. Radenovic, J. Brivio, V. Giacometti, and A. Kis, Nat. Nanotechnol. 6, 147 (2011).

${ }^{10}$ A. A. Balandin, S. Ghosh, W. Bao, I. Calizo, D. Teweldebrhan, F. Miao, and C. N. Lau, Nano Lett. 8, 902 (2008).

${ }^{11}$ J. H. Seol, I. Jo, A. L. Moore, L. Lindsay, Z. H. Aitken, M. T. Pettes, X. Li, Z. Yao, R. Huang, D. Broido, N. Mingo, R. S. Ruoff, and L. Shi, Science 328, 213 (2010).

${ }^{12}$ S. Ghosh, W. Bao, D. L. Nika, S. Subrina, E. P. Pokatilov, C. N. Lau, and A. A. Balandin, Nat. Mater. 9, 555 (2010).

${ }^{13}$ C. Faugeras, B. Faugeras, M. Orlita, M. Potemski, R. R. Nair, and A. K. Geim, ACS Nano 4, 1889 (2010).

${ }^{14}$ H. Sevinçli and G. Cuniberti, Phys. Rev. B 81, 113401 (2010).

${ }^{15}$ J. Haskins, A. Kinaci, C. Sevik, H. Sevinçli, G. Cuniberti, and T. Çă̆ın, ACS Nano 5, 3779 (2011).

${ }^{16}$ A. A. Balandin, Nat. Mater.10, 569 (2011).
${ }^{17}$ J.-W. Jiang, J.-S. Wang, and B.-S. Wang, App. Phys. Lett. 99, 043109 (2011)

${ }^{18}$ C. Sevik, H. Sevinçli, G. Cuniberti, and T. Çağın, Nano Lett. 11, 4971 (2011).

${ }^{19}$ X. Li, W. Cai, L. Colombo, and R. S. Ruoff, Nano Lett. 9, 4268 (2009).

${ }^{20}$ L. Ci, L. Song, C. Jin, D. Jariwala, D. Wu, Y. Li, A. Srivastava, Z. F. Wang, K. Storr, L. Balicas, F. Liu, and P. M. Ajayan, Nat. Mater. 9, 430 (2010).

${ }^{21}$ L. Song, L. Balicas, D. J. Mowbray, R. B. Capaz, K. Storr, L. Ci, D. Jariwala, S. Kurth, S. G. Louie, A. Rubio, and P. M. Ajayan, e-print arXiv:1105.1876v1 (unpublished).

${ }^{22}$ N. Mingo, K. Esfarjani, D. A. Broido, and D. A. Stewart, Phys. Rev. B 81, 045408 (2010).

${ }^{23}$ B. Abeles, Phys. Rev. 131, 1906 (1963).

${ }^{24}$ W. Li, H. Sevinçli, G. Cuniberti, and S. Roche, Phys. Rev. B 82, 041410 (2010).

${ }^{25}$ W. Li, H. Sevinçli, S. Roche, and G. Cuniberti, Phys. Rev. B 83, 155416 (2011).

${ }^{26}$ R. Saito, G. Dresselhaus, and M. S. Dresselhaus, Physical Properties of Carbon Nanotubes (Imperial College Press, London, 1998).

${ }^{27}$ J. Zimmermann, P. Pavone, and G. Cuniberti, Phys. Rev. B 78, 045410 (2008).

${ }^{28}$ C. Sevik, A. Kinaci, J. B. Haskins, and T. Çağın, Phys. Rev. B 84, 085409 (2011).

${ }^{29}$ J. Serrano, A. Bosak, R. Arenal, M. Krisch, K. Watanabe, T. Taniguchi, H. Kanda, A. Rubio, and L. Wirtz, Phys. Rev. Lett. 98, 095503 (2007)

${ }^{30}$ V. N. Popov, Phys. Rev. B 67, 085408 (2003).

${ }^{31}$ L. Wirtz, A. Rubio, R. A. de la Concha, and A. Loiseau, Phys. Rev. B 68, 045425 (2003).

${ }^{32}$ N. Mingo, D. Hauser, N. P. Kobayashi, M. Plissonnier, and A. Shakouri, Nano Lett. 9, 711 (2009).

${ }^{33}$ N. Mingo and D. A. Broido, Nano Lett. 5, 1221 (2005).

${ }^{34}$ L. Lindsay and D. A. Broido, Phys. Rev. B 84, 155421 (2011). 